\title{
Study of prevalence \& degree of sensorineural hearing loss in chronic suppurative otitis media (CSOM)
}

\author{
Chavan P. ${ }^{1}$, Ingale I. ${ }^{2}$, Dixith R. ${ }^{3}$, Anand R. ${ }^{4}$ \\ ${ }^{1}$ Dr. Paresh Chavan, Assistant Professor, ${ }^{2}$ Dr. Mayur Ingale, Assistant Professor, ${ }^{3}$ Rohan Dixith, Resident, ${ }^{4}$ Rohit Anand, \\ Resident; all authors are attached with Department of ENT, Dr D Y Patil Hospital \& Research Centre, DPU, Maharastra, \\ India.
}

Corresponding Author: Dr. Mayur Ingale, Assistant Professor, Department of ENT, Dr. D Y Patil Hospital \& Research Centre, DPU, Maharastra, India. E-mail: dr.mayuringale@gmail.com

\begin{abstract}
Introduction: CSOM is the common chronic infectious disease and is considered the leading cause of acquired hearing loss. Hearing loss is a public health problem in developed and developing countries. Material and methods: A prospective cohort observational study on 100 Cases in two groups of 50 each who had safe CSOM and 50 Unsafe CSOM conducted over a period of July 2011 to September 2013 at Department of Otorhinolaryngology Padmashree Dr. D. Y. Patil Medical College, Hospital and Research Centre, DPU, Pimpri, Pune. Detailed history, complete ENT examination was done. They were then subjected to Puretone audiometry; air and bone conduction thresholds were tested and plotted on the audiogram. Univariate and multivariate logistic regression (odds ratio) analyses were used. Results: In the present study the degree of sensorinueral component of hearing loss was $>35 \mathrm{db}$ in majority of the cases. The mean bone conduction threshold values were obtained by mean value of threshold at frequencies 250, 500, 1000,2000 and $4000 \mathrm{~Hz}$. Higher speech frequencies were affected in majority of patients. Greater sensorineural component was found in CSOM with cholesteatoma. Greater incidence of hearing loss with increasing duration of disease. Conclusion: These findings demonstrate that CSOM is associated with some degree of SNHL and cochlear damage, and higher frequencies are more affected. Increasing age can act as a precipitating factor in this disease process.
\end{abstract}

Keywords- CSOM, Sensoryneural hearing loss, Frequency

\section{Introduction}

Chronic suppurative otitis media (CSOM) is the chronic inflammation of the middle ear fossa with discharge through a perforated tympanic membrane [1]. Hearing loss is a public health problem in developed and developing countries. In the United States, 48.1 million or $20.3 \%$ of the population older than 12 years have unilateral or bilateral hearing loss [2]. Hearing loss causes impaired language development and speech skills in children and poorer life quality and psychological problems for adults [3, 4].

It is acknowledged that size and site of tympanic membrane perforation is proportional to degree of hearing loss but there are many studies which suggests otherwise. Persistent ear discharge is also supposed to deteriorate hearing level with passage of time [5]. This study is carried out to find out the relation between size

Manuscript received: $4^{\text {th }}$ June 2019

Reviewed: $14^{\text {th }}$ June 2019

Author Corrected: $20^{\text {th }}$ June 2019

Accepted for Publication: $26^{\text {th }}$ June 2019 and site of tympanic membrane perforation and duration of ear discharge on hearing loss. Otitis media is the most common otological condition during childhood which compromises sound conduction in the middle ear [6]. In chronic cases, it is estimated that the degree to which hearing is compromised is directly proportional to the damage caused to the middle ear's structures.

It means that hearing thresholds may be influenced by factors such as the size and location of the tympanic perforation, the presence of ossicular chain erosion or disarticulation as well as the presence of cholesteatoma and its growth patterns [6].

The chronic otitis media is defined as a permanent perforation of the drum membrane, which does not close by itself, and an inflammatory reaction in the mucosa (mucositis) of the middle ear. Two main forms of the chronic otitis media are distinct: the suppurative otitis media and the cholesteatoma [7]. 


\section{Original Research Article}

As we can cure the conductive deafness by surgery, the sensorineural deafness is still a challenge to us. Children having mixed loss of hearing invariably suffer from the point of view of education and development of language and therefore it becomes essential to study such cases so that we can prevent the sensorineural deafness in chronic suppurative otitis media.

With this idea in mind, it was decided to study all cases of chronic suppurative otitis media with mixed hearing loss to find out the causes and ways of preventing sensorineural hearing loss in chronic suppurative otitis media. The present study is was $\mathrm{n}$ attempt to study the pattern of hearing loss in CSOM and clinical factors if any that might affect the sensorineural component.

\section{Material and Methods}

The present study is prospective cohort observational study on 100 Cases in two groups of 50 each who had safe CSOM and 50 Unsafe CSOM.

Conducted over a period of July 2011 to September 2013 at Department of Otorhinolaryngology Padmashree Dr. D. Y. Patil Medical College, Hospital and Research Centre, DPU, Pimpri, Pune.

\section{Study design: Observational study}

Inclusion criteria: All CSOM cases with copious ear discharge, central perforation on otoscopy. are included in safe group and All cases with scanty discharge foul smelling, attic or marginal perforation, granulation tissue or cholesteatoma on otoscopy are included in unsafe group.

Exclusion criteria: Age below 1 year and above 50 years, Prior otologicalsurgery, History of head injury, Acoustic trauma, Traumatic tympanic membrane perforation, Systemic ototoxic drugs, Cardiovascular and metabolic disease, Hereditary causes.
Collection of data: Detailed history was elicited; complete ENT examination was done to look for status of otorrhoea, type of perforation, ossicular disruption and presence of granulation tissue or cholesteatoma. They were then subjected to Puretone audiometry; air and bone conduction thresholds were tested and plotted on the audiogram. Narrow band masking was used wherever appropriate. Aural swabs were collected and innoculated for culture and sensitivity.

Operative findings of all cases which underwent surgery were noted. Middle ear effusions at round window were observed. Changes at round window evident under microscope such as obliteration due to bone formation were noted. Presence of labyrinthine erosion or fistula were looked in suspected cases. Cases with labyrinthine fistula found at surgery or with positive fistula sign were documented. The duration of disease was considered on the basis of onset of otorrhoea except in cases of otitis media with effusion.

The mean bone conduction thresholds were obtained for various pathological lesions, by using thresholds at 250, $500,1000,2000$ and $4000 \mathrm{~Hz}$ frequencies. For the predilection, these values were compared with thresholds at 2000 and $4000 \mathrm{~Hz}$. to decide whether lower or higher frequency loss is common. Nonrecordable values of bone conduction were excluded from the data analysis and were used separately for finding out the incidence of total deafness in a particular type of lesion.

Statistical analysis: Continuous variables were described as means (95\% confidence interval) and the difference was observed by using $\mathrm{T}$ test. Differences for categorical variables were assessed by the chi-square test. Univariate and multivariate logistic regression (odds ratio $[\mathrm{OR}]$ ) analyses were used to identify those variables associated with AKI and mortality. $p$ value $<$ 0.05 was considered significant.

\section{Results}

Table No.-1: Incidence of SNHL in different age groups.

\begin{tabular}{|c|c|c|}
\hline Age group of patients & No. of patients with CSOM & Incidence of SN component of hearing loss \\
\hline $11-20$ & 35 & $2(5.71 \%)$ \\
\hline $21-30$ & 25 & $4(16.00 \%)$ \\
\hline $31-40$ & 24 & $5(20.81 \%)$ \\
\hline $41-50$ & 16 & $6(37.50 \%)$ \\
\hline Total & $\mathbf{1 0 0}$ & \\
\hline
\end{tabular}

Incidence of SNHL increases as age increases being maximum in age group of 41-50 years i.e. $37.50 \%$ and minimum in $11-20$ years i.e. $5.71 \%$. 


\section{Table No.-2: Correlation of SNHL component with duration of CSOM}

Mean bone conduction thresholds were deduced at different frequencies and tabulated against duration of CSOM. This study found greater incidence of hearing loss with increasing duration of disease, Hence, the incidence of SNHL component has correlation with duration of disease. $>35 \mathrm{db}$ hearing loss in 9 cases which is maximum 13-30 months

\begin{tabular}{|c|c|c|c|c|c|c|}
\hline & \multicolumn{5}{|c|}{ Duration of CSOM (In months) } & N9-60 \\
\hline Deafness & Upto12 & $\mathbf{1 3 - 2 4}$ & $\mathbf{2 5 - 3 6}$ & $\mathbf{3 7 - 4 8}$ & $\mathbf{4 9}$ of Cases \\
\hline $20-25 \mathrm{~dB}$ & 1 & 0 & 0 & 1 & 0 & 2 \\
\hline $26-30 \mathrm{~dB}$ & 0 & 2 & 0 & 1 & 1 & 4 \\
\hline $31-35 \mathrm{~dB}$ & 0 & 0 & 1 & 1 & 0 & 2 \\
\hline$>35 \mathrm{~dB}$ & 0 & 3 & 3 & 3 & 0 & 9 \\
\hline
\end{tabular}

Table No.-3: Degree of sensorineural hearing loss.

\begin{tabular}{|c|c|}
\hline Bone conduction threshold range & No. of patients with SN component of hearing loss \\
\hline $5 \mathrm{~dB}-10 \mathrm{~dB}$ & - \\
\hline $10 \mathrm{~dB}-15 \mathrm{~dB}$ & - \\
\hline $15 \mathrm{~dB}-20 \mathrm{~dB}$ & - \\
\hline $20 \mathrm{~dB}-25 \mathrm{~dB}$ & 2 \\
\hline $25 \mathrm{~dB}-30 \mathrm{~dB}$ & 4 \\
\hline $30 \mathrm{~dB}-35 \mathrm{~dB}$ & 2 \\
\hline$>35 \mathrm{~dB}$ & 9 \\
\hline
\end{tabular}

In the present study the degree of sensorinueral component of hearing loss was $>35 \mathrm{~dB}$ in majority of the cases

Table No.-4: Incidence of sensorineural component of hearing loss at speech frequencies

The mean bone conduction threshold values were obtained by mean value of threshold at frequencies $250,500,1000$, 2000 and $4000 \mathrm{~Hz}$.

\begin{tabular}{|c|c|}
\hline Frequency $(\mathbf{H z})$ & No. of cases with increased threshold \\
\hline 250 & 2 \\
\hline 500 & 2 \\
\hline 1000 & 3 \\
\hline 2000 & 6 \\
\hline 4000 & 4 \\
\hline
\end{tabular}

In the present study, higher speech frequencies were affected in majority of patients.

Table No.-5: Table showing bone threshold at different frequency in safe and unsafe CSOM.

\begin{tabular}{|c|c|c|}
\hline \multirow{2}{*}{ Bone conduction threshold } & \multicolumn{2}{|c|}{ Chronic suppurative otitis media } \\
\cline { 2 - 3 } & Unsafe CSOM & Safe CSOM \\
\hline$\geq 20 \mathrm{Db}$ in $500 \mathrm{~Hz}, 1000 \mathrm{~Hz}$ & 6 & 1 \\
\hline$\geq 20 \mathrm{~dB}$ in $2000 \mathrm{~Hz}$ & 7 & 3 \\
\hline & 13 & 4 \\
\hline
\end{tabular}

Greater sensorineural component was found in CSOM with cholesteatoma. 
Table No.-6: Type of Pathology.

\begin{tabular}{|c|c|}
\hline Pathology & No. of cases \\
\hline SAFE CSOM: & 50 \\
\hline Perforation: & 12 \\
\hline Small & 18 \\
\hline Medium & 20 \\
\hline Subtotal & 50 \\
\hline UNSAFE CSOM: & 19 \\
\hline Marginal perforation & 31 \\
\hline Attic pouch / perforation & \\
\hline
\end{tabular}

Above studies, shows that perforation in safe CSOM are 12 small, 18 medium, 20 subtotal and in unsafe CSOM 19 marginal, 31 attic

\section{Discussion}

Sensorineural loss, either alone or with conductive loss may occur in acute as well as chronic suppurative otitis media. 100 cases of chronic suppurative otitis media examined clinically and with aid of audiometry showed 17 patients with mixed hearing loss.

Bone conduction thresholds were used to define the degree of sensorineural hearing loss. In the present study sensorineural hearing loss was defined as bone conduction loss of $20 \mathrm{~dB}$ or more at frequencies $250 \mathrm{~Hz}$, $500 \mathrm{~Hz}, 1000 \mathrm{~Hz}, 2000 \mathrm{~Hz}$ and $4000 \mathrm{~Hz}$. The degree of hearing loss was $>35 \mathrm{db}$ in majority of the patients. This is consistent with findings ospapp $\mathrm{z}$ et al [8].

The files of 121 patients with unilateral chronic suppurative otitis media were reviewed in a retrospective study. Air conduction and bone conduction threshold averages were calculated over the speech frequencies $(500 \mathrm{~Hz}, 1,000 \mathrm{~Hz}$, and 2,000 Hz). Thresholds at $4 \mathrm{kHz}$ were examined separately but in a similar way. Chronic suppurative otitis media was seen to be associated with sensorineural hearing loss.

When age and normal side were corrected for, pure-tone threshold and bone conduction threshold at either the speech frequencies or at $4 \mathrm{kHz}$ increased gradually according to the duration of the chronic suppurative otitis media. The threshold shift was more accentuated as age increased. The sensorineural hearing loss at 4 $\mathrm{kHz}$ seemed to be higher than that at the speech frequencies $[8,9,10,11]$.

Kholmatov DI.et al[12] found that To detect initial signs of internal ear dysfunction in 87 patients with otitis media purulentachronica (OMPC), wide-rage frequency audiometry (WRFA) in bone and air conduction according to B.M. Sagalovich was used. Most of the patients with normal bone conduction in frequencies up to $8 \mathrm{kHz}$ had higher bone thresholds in wide-range frequencies. In mixed hypoacusis these thresholds rose to the highest degree. A correlation was found between a rise of auditory thresholds wide range of frequencies in bone conduction and the disease duration.

In this series, it has been clinical impression that SNHL does occur in CSOM with particular involvement of higher frequencies similar to the study by Kamaljit Kaur et al [12]. An incidence of $24 \%$ of SNHL was detected in 100 cases of unilateral CSOM. Although the work of many eminent investigators has supported this view, there are others who deny an association between CSOM and SNHL.

When the duration of disease was compared with the incidence of SNHL, a progressively increasing incidence of SNHL was found as the duration of disease increased. The incidence of SNHL was found to be $13.64 \%$ when the duration of disease was $<5$ year and progressively rose to $33.33 \%$ when the duration of disease was $>26$ years. Similar correlation between duration of disease and SNHL has been reported by Kholmatov (2001) [13]. However, Noordzig et al (1995) [14] and Mac Andie et al (1999)[15] did not find a correlation between SNHL and duration of CSOM.

Jesic SD et al [16] Mean values of bone conduction thresholds for frequencies 512 to $4,096 \mathrm{~Hz}$ were significantly higher in otitis groups than in healthy ears $(p=0.000)$, without differences between the groups. 
Ossicular disruption correlated with sensorineural hearing loss in cholesteatoma and tubotympanic otitis for all frequencies; long incus process destruction in tubotympanic ears showed strong negative correlation with sensorineural hearing loss for 512 to $2,048 \mathrm{~Hz}$ (linear regression coefficient, intercept was $-2.84,-2.48$, and $-2.41 ; \mathrm{p}=0.0024,0.0207$, and 0.0076 , respectively).

Perforation size correlated with sensorineural hearing loss for 512 to $2,048 \mathrm{~Hz}$ in tubotympanic otitis ( $\mathrm{Log}$ regression $\mathrm{p}=0.0008,0.0252$, and 0.0267 ; odds ratio, $1.13,1.11$, and 1.06). Atelectasis correlated with sensorineural hearing loss for $4,096 \mathrm{~Hz}(\mathrm{p}=0.022)$.

In another study, Silveira Netto LF et al [6] Orji FT et al [9] found that Air conduction, bone conduction thres holds and air-bone gaps in children and teenagers with CCOM are significantly greater.

There were no significative differences between airbone gaps in epitympanic and posterior mesotympanic cholestea-tomas. In NCCOM, the gap value is positively correlated with the number of quadrants with tympanic perforation.

There was no significative difference between the airbone gaps in tympanic perforations affecting the posterior and anterior quadrants. Redaelli de Zinis LO et al [10] found that selected clinical features were assessed among diseased ears to examine possible influences on inner ear function. Mean bone conduction threshold differences varied from $0.6 \mathrm{~dB}$ at $0.5 \mathrm{kHz}$ to $3.7 \mathrm{~dB}$ at $4 \mathrm{kHz}$.

These differences augmented with increasing duration of middle ear disease. Impaired hearing by bone conduction thresholds of diseased ears correlated with increased age at every frequency and with an interruption of the ossicular chain only at higher frequencies.

The severity of sensorineural hearing loss correlated with longer duration of middle ear disease. Thus, surgical treatment of dry and apparently stable tympanic membrane perforation is warranted.

Papp $\mathrm{Z}$ et al [8] found that chronic suppurative otitis media was seen to be associated with sensorineural hearing loss. When age and normal side were corrected for, pure-tone threshold and bone conduction threshold at either the speech frequencies or at $4 \mathrm{kHz}$ increased gradually according to the duration of the chronic suppurative otitis media. The threshold shift was more accentuated as age increased.
The inner and middle ear are connected mainly through the round and oval windows, and these membranes may allow several toxins to pass from the middle ear into the inner ear. This implies that disturbances of the inner ear could be caused by middle ear inflammation [17].

In this sense, control of middle ear inflammation in the early stage is essential to decreasing the risk of inner ear disturbances [17].

Despite advances in public health and medical care, chronic suppurative otitis media is still prevalent around the world. It is most common in developing countries and in certain high risk populations in developed nations, as well as among children who have tympanostomy tubes inserted.

Since this chronic infection is caused by persistent acute otorrhea, which in turn is usually secondary to acute otitis media, prevention should be directed toward prompt and appropriate treatment of the acute middleear infection $[18,19]$.

Repair of chronic perforations should prevent recurrence, since reinfection is due either to reflux of pathogenic organisms from the nasopharynx into the middle ear, or water contamination from the external canal. Information from epidemiological studies, which show that populations can be categorized into highest, high, low and lowest prevalence, can be helpful in setting national priorities for prevention and treatment $[19,20]$.

In the light of this literature review, there is a need for the continual assessment of sensorineural function in patients with CSOM. When SNHL develops, active surgical or medical intervention should be considered if not already done.

Too many physicians in medicine consider CSOM to be an innocuous process unless an obvious complication develops.

\section{Conclusion}

CSOM is associated with mixed hearing loss mainly $>35 \mathrm{~dB}$, higher frequencies were involved. There was no significant correlation between degree of sensorineural hearing loss and duration of otorrhoea. It was also observed that the incidence of SNHL increased with the increasing duration of disease.

More audiologic studies in patients with CSOM are needed to evaluate the sensoryneural component of 


\section{Original Research Article}

hearing loss it would be interesting if such studies included the entire upper frequency spectrum since it is possible that very high frequency losses occur which are not perceptible to the patient and are not measured by routine audiometery.

A high frequency audiometry test (i. e. $10,000 \mathrm{~Hz}$ to $20,000 \mathrm{~Hz}$ ) is advisable in all patients of CSOM and it is emphasized that the cases of chronic suppurative otitis media should be diagnosed early, and managed effectively so as to prevent the chances of developing sensorineural hearing loss.

\section{Funding: Nil, Conflict of interest: Nil \\ Permission from IRB: Yes}

\section{References}

1. Amali A, Hosseinzadeh N, Samadi S, Nasiri S, Zebardast J. Sensorineural hearing loss in patients with chronic suppurative otitis media: Is there a significant correlation? Electron Physician. 2017;9(2):3823-3827. DOI: 10.19082/3823. eCollection 2017 Feb.

2. Lin FR, Niparko JK, Ferrucci L. Hearing loss prevalence in the United States. Arch Intern Med. 2011; 171 (20): 1851-2. DOI: 10.1001/ archinternmed. 2011.506.

3. Jensen RG, Koch A, Homøe P. The risk of hearing loss in a population with a high prevalence of chronic suppurative otitis media. Int J Pediatr Otorhinolaryngol. 2013;77(9):1530-5. DOI: 10.1016/j.ijporl.2013.06.025. Epub 2013 Jul 29.

4. Bakir S, Kinis V, Bez Y, Gun R, Yorgancilar E, Ozbay M, Aguloglu B, Meric F. Mental health and quality of life in patients with chronic otitis media. Eur Arch Otorhinolaryngol. 2013;270(2):521-6. DOI: 10.1007/s00405-012-2031-6. Epub 2012 May 8.

5. Maharjan M, Kafle P, Bista M, Shrestha S, Toran KC. Observation of hearing loss in patients with chronic suppurative otitis media tubotympanic type. Kathmandu Univ Med J. 2009;7(4):397-401.

6. Silveira Netto LF1, da Costa SS, Sleifer P, Braga ME.The impact of chronic suppurative otitis media on children's and teenagers' hearing. Int $\mathrm{J}$ Pediatr Otorhinolaryngol. 2009;73(12):1751-6. DOI: 10.1016/j. ijporl. 2009.09.033. Epub 2009 Oct 23.

7. Reiß M, Reiß G. Suppurative chronic otitis media: etiology, diagnosis and therapy. Med Monatsschrift fur Pharma. 2010;33(1):11-6.
8. Papp Z, Rezes S, Jókay I, Sziklai I. Sensorineural hearing loss in chronic otitis media. Otol Neurotol. 2003;24 (2):141-4.

9. Orji FT, Agu CC. Patterns of hearing loss in tympanic membrane perforation resulting from physical blow to the ear: a prospective controlled cohort study. Clinical Otolaryngol. 2009;34(6):526-32.

10. Redaelli de Zinis LO, Campovecchi C, Parrinello G, Antonelli AR. Predisposing factors for inner ear hearing loss association with chronic otitis media: Factores predisponentes en la asociación de la hipoacusia del oÍdo interno con la otitis media crÓnica. Int $\mathrm{J}$ Audiology. 2005;44(10):593-8.

11.Babić B, Arsović N. Assessment of senses of hearing and balance in chronic suppurative otitis media. Srp Arh Celok Lek. 2008;136(5-6):307-12.

12.Kaur K, Sonkhya N, Bapna AS. Chronic suppurative otitis media and sensorineural hearing loss: Is there a correlation?. Indian J Otolaryngol Head Neck Surg. 2003;55(1):21-4.

13. Kholmatov DI. Early detection of a sensorineural aspect of hypoacusis in patients with chronic purulent otitis media. Vestn Otorinolaringol. 2001;(3):26-8.

14. Noordzij JP, Dodson EE, Ruth RA, Arts HA, Lambert PR. Chronic otitis media and sensorineural hearing loss: is there a clinically significant relation? Am J Otol. 1995;16(4):420-3.

15. MacAndie C, O'Reilly BF. Sensorineural hearing loss in chronic otitis media. Clin Otolaryngol Allied Sci. 1999;24(3):220-2. DOI: https://doi.org/10.1046/j. 1365-2273.1999.00237.x

16. Jesic SD, Jotic AD, Babic BB. Predictors for sensorineural hearing loss in patients with tubotympanic otitis, cholesteatoma, and tympanic membrane retractions.Otol Neurotol. 2012; 33 (6):934-40. doi: 10.1097/ MAO. 0b013e318259b885.

17. Sone M. Inner ear disturbances related to middle ear inflammation. Nagoya J Med Sci. 2017;79(1):1-7. DOI: 10.18999/nagjms.79.1.1.

18. Liberman MC, Liberman LD, Maison SF. Chronic Conductive Hearing Loss Leads to Cochlear Degeneration. PLoS One. 2015 Nov18; 10 (11): e014 2341 DOI: 10.1371/journal. pone. 0142341. eCollection 2015. 
19. Bluestone CD. Epidemiology and pathogenesis of chronic suppurative otitis media: implications for prevention and treatment. Int $\mathrm{J}$ Pediatr Otorhinolaryngol. 1998;42(3):207-23.
20. Mizutari K. Blast-induced hearing loss. J Zhejiang Univ Sci B. 2019; 20(2):111-115. DOI: 10.1631/jzus. B1700051. Epub 2017 Oct 18.

\section{How to cite this article?}

Chavan P, Ingale I, Dixith R, Anand R. Study of prevalence \& degree of sensorineural hearing loss in chronic suppurative otitis media (CSOM). Trop J Ophthalmol Otolaryngol.2019;4(2):163-169.doi:10.17511/jooo.2019.i02.16 